\title{
La cultura del departamento en la modernidad: el caso de estudio del multifamiliar en CU-UNAM.
}

Modern apartment culture: multifamily housing at CU-UNAM, a case study.

Recibido: febrero/2018

Aceptado: agosto/2018

\section{Resumen}

El multifamiliar para maestros de la UNAM es una singular realización de M. Pani y S. Ortega de los años cincuenta. Fue su tercer proyecto de arquitectura habitacional colectiva y en densidad tras la experiencia de las unidades multifamiliares más conocidas (CUPA y CUPJ). Su origen se remonta a una planeación urbana y a una problemática específica como fue la fundación de un ambicioso proyecto del México moderno: la Ciudad Universitaria de la UNAM. Por medio de un estudio de la documentación disponible en archivos y revistas, se ha problematizado y contextualizado la génesis, morfología y técnica proyectual de este caso de estudio, en el cual se observa el desafío de crear un modelo de habitación "moderna" vertical, en una dificultosa lucha por convencer a una población apegada a otra cultura doméstica. Las propuestas iniciales, basadas en estructuras espaciales que reproducen y/o emulan organizaciones como el de la casa unifamiliar, llevan a concluir un proyecto, con cierto espíritu pionero, en el que se consigue crear un inmueble de viviendas en duplex que recuerdan, sutilmente, la distribución de la casa familiar ancestral.

\section{Palabras Clave:}

Arquitectura mexicana moderna; Vivienda multifamiliar; M. Pani.

\author{
Alejandro Leal Menegus ${ }^{1}$ \\ Alejandro Pérez Duarte ${ }^{2}$ \\ Bruno Cruz Petit ${ }^{3}$
}

\begin{abstract}
The multifamily-housing for UNAM teachers is a singular work by M. Pani and S. Ortega belonging to their the 1950's period. It was their third project dealing with high density residential architecture, after the experience of their most known dwelling units (CUPA and CUPJ). Its origin goes back to an urban planning and to a specific problem as it was the foundation of an ambitious project of the modern Mexico: UNAM Ciudad University City. Through a study of the documentation available in archives and journals, we problematize and contextualize the genesis, morphology and design technique of this case in which the challenge of creating a vertical "modern" building is observed in relation to a struggle to convince a population attached to another domestic culture. The initial proposals, based on spatial structures that reproduce and / or emulate organizations such as the single family home led to a project with a certain pioneering spirit, in which it was possible to create a duplex housing building that subtly recalled the distribution of the ancestral family house.
\end{abstract}

\section{Keywords:}

Modern mexican architecture; Multifamily

housing; M. Pani.

\footnotetext{
${ }^{1}$ Centro de Investigaciones en Arquitectura Urbanismo y Paisaje, Facultad de Arquitectura, Universidad Nacional Autónoma de México; Doctor en Arquitectura. arq.leal@gmail.com

${ }^{2}$ Universidade Fumec (Brasil). Doctor en Arquitectura. apdf230174@live.com

${ }^{3}$ Universidad Motolinía del Pedregal (México). Doctor en Sociología. cruzpetit@hotmail.com
} 


\section{El problema de la habitación universitaria.}

El multifamiliar de M. Pani y S. Ortega para la UNAM ha sido analizado de manera relativamente marginal en comparación con las obras más conocidas del equipo de arquitectos realizadas durante sexenio del Presidente M. Alemán (1948-1952). Alba Martínez (1993) le dedica unas pocas líneas; De Anda (2008: 268) ve en él la confirmación del modelo de multifamilar trabajado en los años anteriores, con un paralelepípedo "adecuado para resolver orientación, iluminación, ventilación y vistas al exterior"; L. Noelle (2008: 143) lo describe como "un prototipo de lo que se pensaba que fuera toda una unidad habitacional para los profesores de la magna casa de estudios (...) y por tratarse de una vivienda, se encuentra situado fuera del campus escolar"; Gómez Porter (2016) no lo incluye en su artículo sobre los multifamiliares de M. Pani. Y, si bien la magnitud de la obra no es la misma que la de las unidades habitacionales previas promovidas por la Dirección General de Pensiones, a nuestro juicio, el edificio tiene un interés indudable, al insertar el debate sobre la arquitectura habitacional moderna en la dinámica más amplia de lo que fue una de las grandes aportaciones de la modernidad alemanista: la construcción de Ciudad Universitaria, en el sur de la Ciudad de México.

Figura 1. Multifamiliar para maestros de Ciudad Universitaria. 1953.

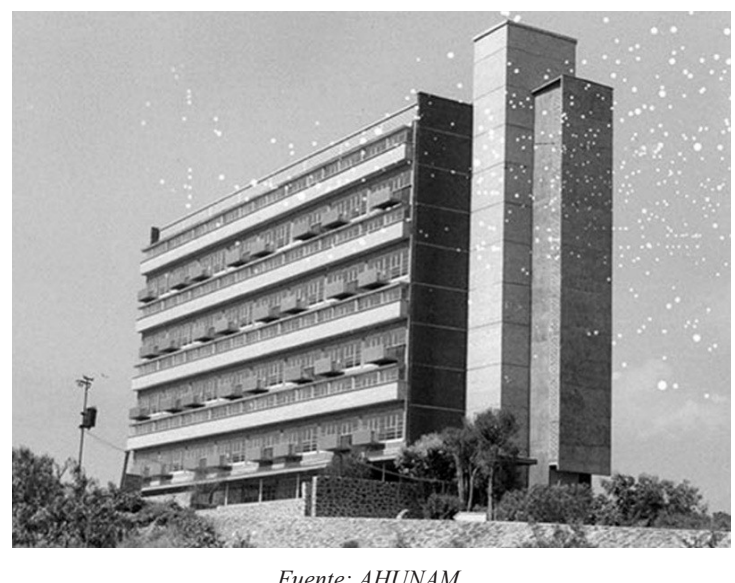

Desde la autonomía universitaria en 1929 uno de los proyectos más ambiciosos de la Universidad había sido reunir en un nuevo campus todas sus dependencias con el propósito de asumir el reto creciente que la masa demográfica y la importancia de los estudios universitarios representaban para el desarrollo del país y de la propia institución. Este ideal se vio reflejado durante el rectorado de Ignacio García Téllez (1929-1932) quien dedicó tiempo y esfuerzo a dicho propósito. Con el modelo de "ciudad universitaria" europeo en mente, se concibió unir el tema habitacional al programa del proyecto de la ciudad universitaria en el Pedregal de San Ángel. El rector había expresado su voluntad de que el campus incluyera pabellones (habitacionales) para alumnos extranjeros. E incluso se adquirieron los terrenos para el proyecto (257 ha). Los terrenos eran colindantes a la colonia Chapultepec Heights y se encontraban en los límites entre el Estado de México y el Distrito Federal., Sin embargo, el campus finalmente no se construyó y en 1937 se vendieron los terrenos.

La decisión de afincar el nuevo campus en las Lomas de San Isidro y San Joaquín o después, como ocurrió finalmente, en el Pedregal de San Ángel, obedeció al hecho de haber adquirido una cantidad importante de terreno urbanizable en las cercanías de la Ciudad de México en un buen precio. Es decir, la ciudad universitaria tendría forzosamente una relación periférica o suburbana con respecto a la Ciudad de México. Desde el primer intento en la década de 1930, se vio esta circunstancia como un problema potencial, pues tanto los profesores, como los alumnos habitaban y trabajan en distintas partes de la ciudad y la distancia podía generar un descontento (Sánchez (2014: 99).

Para principios de la década de 1950 , conforme se fue haciendo tangible la Ciudad Universitaria (CU) en el Pedregal de San Ángel, la discusión en torno a su lejanía comenzó a recobrar fuerza ${ }^{4}$. Tanto trabajadores administrativos como académicos vieron la posibilidad de hacerse de una vivienda en lo que en efecto sería una nueva ciudad.

La extensión del terreno y la magnitud de las obras alimentaban este espíritu, el cual pro-

\footnotetext{
${ }^{4}$ Cuestión que el arquitecto Carlos Obregón Santacilia había recalcado unos años antes en el escrito que redacto cuando formó parte de la Comisión interministerial de la Ciudad Universitaria en 1946 donde señaló las ventajas e inconvenientes de la construcción de CU en el pedregal de San Ángel.
} 
gresivamente se convirtió en un instrumento político; se llegó a condicionar la mudanza física del personal a las nuevas instalaciones si no se resolvía el tema habitacional (Pérez-Méndez $(2014 ; 82)$. De ahí que se formara una Comisión coordinadora del traslado a CU para dicho efecto y que se justificara la rápida construcción del multifamiliar "muestra" para maestros. Paralelamente también se propuso y luego se descartó la habitación para estudiantes, pues finalmente la política del Estado fue fomentar las universidades estatales y no promover el traslado de estudiantes del interior del país a la capital. Pese a ello, el anterior punto estaba vigente al seno de la universidad y el rector inclusive comisionó al arquitecto Enrique del Moral para que estudiara el asunto en un viaje que hizo a Europa en $1953^{5}$.

La incorporación de los empleados universitarios a la federación de sindicatos de trabajadores al servicio del Estado posibilitó que éstos pudieran acceder a la vivienda promovida por la Dirección de Pensiones Civiles y ser inquilinos de los Centros Urbanos Multifamiliares, como el presidente Juárez. La distancia del Pedregal de San Ángel respecto a la ciudad en ese momento hacía en principio más atractiva la opción de un conjunto habitacional en CU.

El único edificio habitacional que finalmente se edificó en Ciudad Universitaria, conocido como el Multifamiliar para Maestros, fue construido entre 1951 y 1952 por la compañía In- genieros Civiles Asociados (ICA), empresa que había levantado los Centros Urbanos Presidente Alemán y Presidente Juárez. La Dirección de Pensiones Civiles fue la institución a cargo de contratar a precio alzado ${ }^{6}$ la construcción del proyecto de los arquitectos Mario Pani y Salvador Ortega en un predio de poco más de una hectárea ${ }^{7}$ dentro de los extensos terrenos de la Ciudad Universitaria (730 ha) en el Pedregal de San Ángel. ${ }^{8}$ El terreno cedido a dicha institución para este propósito se ubicó al poniente del conjunto, en lo que en aquel momento se denominó como "Fraccionamiento para maestros". Curiosamente, dicho fraccionamiento sólo se compuso de un multifamiliar y no contó con un área comercial. ${ }^{9}$ En otras palabras, fue un multifamiliar desprovisto del concepto de "centro urbano", circunstancia que desde su origen condicionó la forma en que se habitó y que lo diferencia del resto de multifamiliares de pensiones construidos a mediados del siglo XX en la Ciudad de México.

Los otros proyectos de habitación en Ciudad Universitaria, la denominada "Unidad tipo habitación" a cargo de los arquitectos Enrique Carral Icaza y Manuel Martínez Páez, así como el "Edificio del Departamento del Distrito Federal para habitaciones de estudiantes" de los arquitectos Jorge L. Medellín, Antonio Serrato, Jorge Martin Cadena y el Ing. Roberto Medellín no se realizaron.

\footnotetext{
${ }^{5}$ Visitó Oxford, Paris, Bolonia y Madrid, en donde averiguó no solo aspectos arquitectónicos, sino principalmente cuestiones administrativas, las cuales sintetizo en el memorándum. Ver AHUNAM, Fondo UNAM, Secretaria General, caja 324, expediente 1833, 1953-octubre (De la Asociación de profesores universitarios al rector Dr. Nabor Carrillo). "Memorándum sobre el problema de organización de las Habitaciones de Estudiantes en la CIUDAD UNIVESITARIA".

${ }^{6}$ En el contrato de obra se especifica el costo total en: $\$ 1,298,541.86$ pesos, al considerar como precio Zbase los $\$ 230$ pesos por m2 de construcción del Centro Urbano Presidente Juárez más \$38.42 pesos por m2 más por “diferencias en sus características" y "mejoras en sus especificaciones." Con lo cual, se constata, que más allá del parecido arquitectónico, el costo por m2 de obra fue muy cercano. También, se especifica dentro las cláusulas del contrato que la obra tiene como fecha de terminación el 31 de octubre de 1952, lo que seguramente tuvo que ver con la fecha pactada para la Ceremonia de Dedicación de la Ciudad Universitaria por el presidente Miguel Alemán, la cual ocurrió el 20 de noviembre de 1952. Ver AHUNAM, Fondo UNAM, Secretaria General, caja 324, expediente 1833, 1951-noviembre (Entre la Dirección de Pensiones Civiles e ICA). "Contrato de obra a precio alzado."

${ }^{7}$ La superficie cedida a la Dirección de Pensiones Civiles para construcción del multifamiliar fue de: 11,161.60 m2 . Ver AHUNAM, "Contrato de obra a precio alzado. En contraste, en el libro Los multifamiliares de pensiones se señala un predio de solo 6000m2, es decir la mitad, de la cual, el desplante del edificio ocupa 1,150 m2, quedando el 80\% como área libre (Pani,1952, p.109).

8 Curiosamente en el contrato se señala como un terreno "anexo" a la Ciudad Universitaria, sin embargo, el emplazamiento del multifamiliar y el fraccionamiento de maestros, no colinda con el límite del terreno de CU. El documento precisa que lo acompaña un plano de ubicación del terreno, sin embargo, este no está anexado al documento que se encuentra en el archivo. Ver AHUNAM, "Contrato de obra a precio alzado."

${ }^{9}$ En la Dirección General de Obras y Conservación de la UNAM se resguarda un plano de anteproyecto fechado en septiembre 1950 y firmado por Mario Pani Arq. intitulado "C.U. Edificio para profesores". El cual es muy semejante al proyecto que se construyó, salvo por dos cuestiones, a los departamentos dúplex se accede por la planta alta y se baja a la recamaras (al revés de cómo se construyó) y toda la planta baja del edificio está destinada a comercios. Coincidentemente en el "Contrato de obra a precio alzado" se indica que el multifamiliar constará de 32 departamentos dúplex, siendo que en realidad se edificaron 42, 10 más. Teniendo los departamentos restantes un solo nivel, en Arquitectura México núm. 39 los describen como tipo "Alcoba".
} 
Figura 2. Plano del anteproyecto del multifamiliar para maestros de la UNAM en Ciudad Universitaria. 1950.

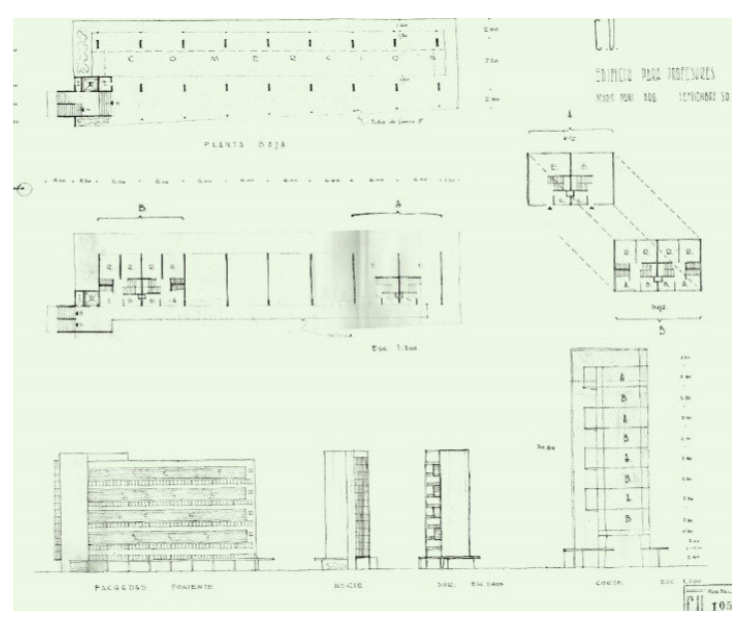

Fuente: Dirección General de Obras y Conservación, UNAM.

Figura 3. Concurso casa habitación del maestro univesitario, proyecto del Arq. Ernesto Gómez Gallardo. 1951.

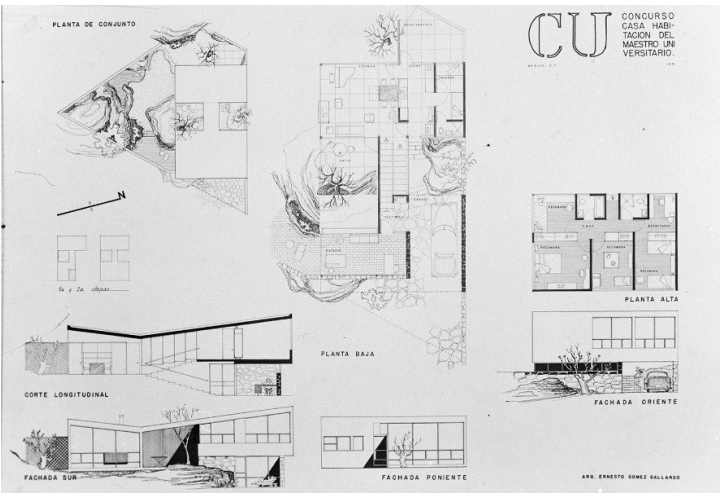

Fuente: AHUNAM.

Una de las razones más importantes detrás de esta situación fue que en algún momento la Comisión de la Ciudad Universitaria (después Comisión Intersecretarial) consideró financiar la construcción de CU con la venta de parte de sus terrenos, circunstancia que no tuvo lugar (Sánchez, 2014:146). Pese a ello, se sentó un precedente en cuanto a la posibilidad de real de volver a vender los terrenos que habían sido fruto de una expropiación o adquirir más terrenos colindantes a la propia universidad para resolver las necesidades de sus trabajadores. La Sociedad de Arquitectos Mexicanos realizó en 1951 el concurso "Casa del Maestro Universitario" (concurso organizado por Carlos Lazo y Almiro Morantinos en colaboración con la SAM; Pérez-Méndez, 2014:110$111)$, en el que quedó patente que el modelo de vivienda óptimo para el catedrático era uno más cercano a las casas en construcción en Jardines del Pedregal que un apartamento dentro de un centro urbano. Como vemos en el proyecto del arquitecto Ernesto Gómez Gallardo la casa se estructura en dos zonas claramente diferenciadas publico/privada, divididas por una diferencia de nivel (figura 5).

Figura 4. Multifamiliar para maestros: corredor de la fachada poniente de los apartamentos tipo "alcoba", actualmente modificada. 1953.

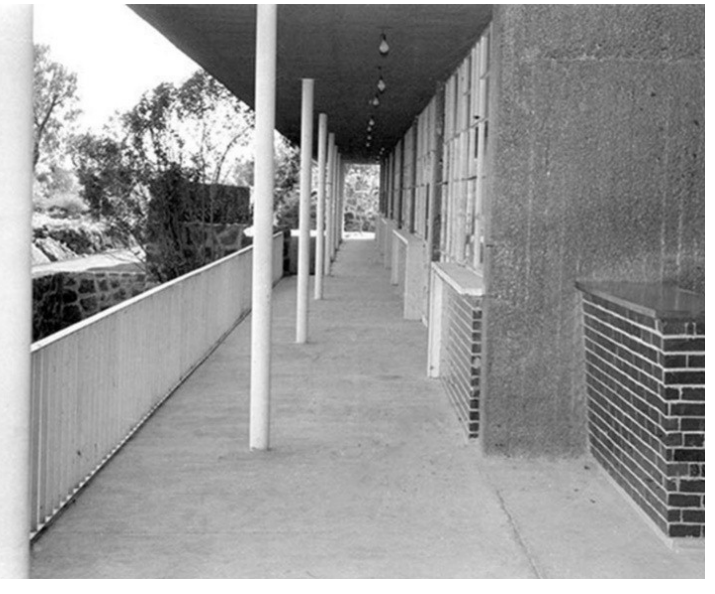

Fuente: AHUNAM

La Ciudad Universitaria en el Pedregal, que se fue construyendo desde 1948, fue inaugurada en 1952 por el presidente Miguel Alemán. Los estudiantes no empezaron clases hasta la primavera de 1954, continuándose los trabajos del proyecto inicial hasta 1958 , pese a que diversas partes de dicho proyecto no se llevaron a cabo, como fue el caso del centro cívico y las unidades de habitaciones para maestros y alumnos. La excepción fue un único multifamiliar con 42 viviendas, el cual se empezó habitar en 1955. Todo ello dejó como resultado que el problema general de la habitación finalmente no se resolvió dentro de Ciudad Universitaria ${ }^{10}$ y un edificio aislado de 42 departamentos.

\footnotetext{
${ }^{10}$ En 1966 desde la Escuela Nacional de Arquitectura, de la mano de su director el Arq. Ramón Torres se realizó un ambicioso proyecto habitacional para maestros dentro de los límites de Ciudad Universitaria, sin embargo, tampoco vio la luz.
} 


\section{Discusiones, avances y dificulta- des para un modelo de habitación familiar.}

El desafío constructivo del Multifamiliar para M. Pani y S. Ortega fue crear una nueva forma habitacional dentro de las ambiciones y espíritu "moderno" del proyecto para la Ciudad Universitaria. No se trataba simplemente de reproducir un modelo tradicional de vivienda, sino que, en nombre de la modernidad, se buscaba la experimentación y la innovación, estando las posibilidades abiertas para cualquier propuesta que no fuese tradicional. La "cultura de la modernidad" entendía que debía ser introducida una nueva forma habitacional, siguiendo los ideales de economía y eficiencia para "una pronta conjuración de la crisis de la vivienda" (Revista Arquitectura/México, 1929, p. 263). Por ejemplo, del CUPA-Conjunto Urbano Presidente Alemán, construido en 1948, se decía en la revista Arquitectura/México que representaba "el esfuerzo más vigoroso llevado al cabo en México [sic] para la resolución del problema de la vivienda", con un "mejor aprovechamiento del terreno", atendiendo las "condiciones de amplitud, confort y comodidad de las viviendas" (Arquitectura México: 267). En contraposición con el entusiasmo anterior, la cultura popular recibió con una mezcla de asombro y consternación aquello que se proponía, se llegó a calificar al CUPA de "campo de concentración" (Acevedo Escobedo cit. en Adriá, 2005: 17), de "palomar, caja de zapatos, grilleras, y otros [apodos] que se apoyaban en la creencia de que al mexicano le gusta más su casita con su terrenito". Enrique del Moral (1964: 11) explica elocuentemente el problema cultural: Frecuentemente se escuchan discusiones respecto a las ventajas o desventajas que tienen las habitaciones multifamiliares y por regla general la gente se inclina-sobre todo en nuestro medio- a preferir la casa unifamiliar. Al llegar a esta conclusión, si hay un arquitecto en el grupo, se tien- de a reprocharle el pecado de haber proyectado edificios multifamiliares. El reproche y la preferencia personal de quienes han argumentado son inoperantes, ya que la solución de construir habitaciones multifamiliares no es un capricho personal, ni del arquitecto ni de quien le encargo la obra esta impuesta por el programa de época. Así, el problema que enfrenta en el proyecto para el Multifamiliar de CU en los años cincuenta se inserta en el tramo final de una década de profundas y acaloradas discusiones sobre el modelo doméstico ideal ${ }^{11}$. En este sentido, la propuesta del multifamiliar en $\mathrm{CU}$ debe ser entendida no solo como un ejercicio de carácter experimental sino como una conclusión de una década de experiencia en el despacho de M. Pani con un objetivo claro: convencer a una cultura tradicional popular de las ventajas de una vivienda, compacta, funcional y verticalizada. ${ }^{12}$

\section{Los dúplex del Multifamiliar de CU y el planeamiento "en sección".}

Una de las estrategias para lograr el anterior objetivo fue incluir en el edificio (de metraje superior al CUPA, seguramente por la pretensión de ofrecer un estatus de acuerdo al perfil de los catedráticos universitarios) un modelo de departamento que, alguna forma, incorporaba la morfología de la casa unifamiliar en un edificio multifamiliar: el dúplex o departamento de dos pisos, un modelo ampliamente estudiado por los arquitectos en la década de los años cincuenta. Por consiguiente, para entender la conspicua propuesta de la célula dúplex para el Multifamiliar de CU es conveniente tener en cuenta algunos antecedentes y las atrevidas propuestas que, en esta línea de trabajo morfológico, estaban puestas en práctica en otras partes del mundo.

M. Pani estaba al corriente de novedades arquitectónica como el edificio Palace Gate (Londres, 1937-39), proyecto de W. Coates, reseñado intensamente en varias revistas de la época como

\footnotetext{
11 "La unión de la sala con el comedor no es grata al mexicano, por un íntimo sentido aristocrático que no permite la unión de estos dos ámbitos y porque la comunión social la realiza en la mesa" (Larrosa, 1985: 98). Según Manuel Larrosa (1985: 161) "la idea de promover este tipo de propiedad por pisos (en condominio) surgió a raíz de un viaje a Sao Paulo, Brasil, donde en 1952 fungió como jurado de la Primera Bienal de Arquitectura y conoció de primera mano la experiencia brasileña."

${ }_{12}$ Tiempo después Pani será el impulsor de la ley de condominio, aplicado por primera vez en su propuesta en Av. Paseo de la Reforma y Guadalquivir: un complejo sistema de organización interna que puede competir con el sistema de privacidad de una casa tradicional (Pérez-Duarte, 2005).
} 
Architectural Record y Architectural Forum, que desafiaba las formas tradicionales de los departamentos bajo una nueva premisa proyectual: el planeamiento en sección. Se trata de una técnica proyectual que consiste en desarrollar la solución a partir de una vista en corte transversal del bloque laminar, y no solo bidimensionalmente desde un estudio de una "planta tipo" (Architectural Record, agosto 1937). Concretamente, el edificio Palace Gate aplicó una forma de organización que Coates llamó 3-2 system, y que dejó gran impacto en Pani, el cual no dudó en publicar inmediatamente una reproducción del proyecto en uno de los primeros números de la revista Arquitectura, de la cual era editor. Cabe mencionar que en el artículo del número de Arquitectura/México de julio 1939 incluyó un complejo dibujo en isométrico de las "circulaciones y accesos" del Palace Gate. La autoría de Pani de dicho dibujo (o el desarrollo bajo su supervisión) evidencia su esfuerzo por entender la técnica, denotando particular interés la capacidad de superar circulaciones entre servicio/propietarios. Y también el interés por la posibilidad de segregar circulación de servicio y de propietarios y poder evitar cruzamientos indeseados. De hecho, la segregación servicio/propietarios es contemplada por arquitectos contemporáneos como Francisco J. Serrano (Pérez Duarte, 2005) y Pani la llega a resolver de forma hábil en el condominio de Av. Paseo de la Reforma y Guadalquivir (Ciudad de México, 1956).

Figura 5. Explicación del sistema de circulaciones de los apartamentos "Tres en dos".

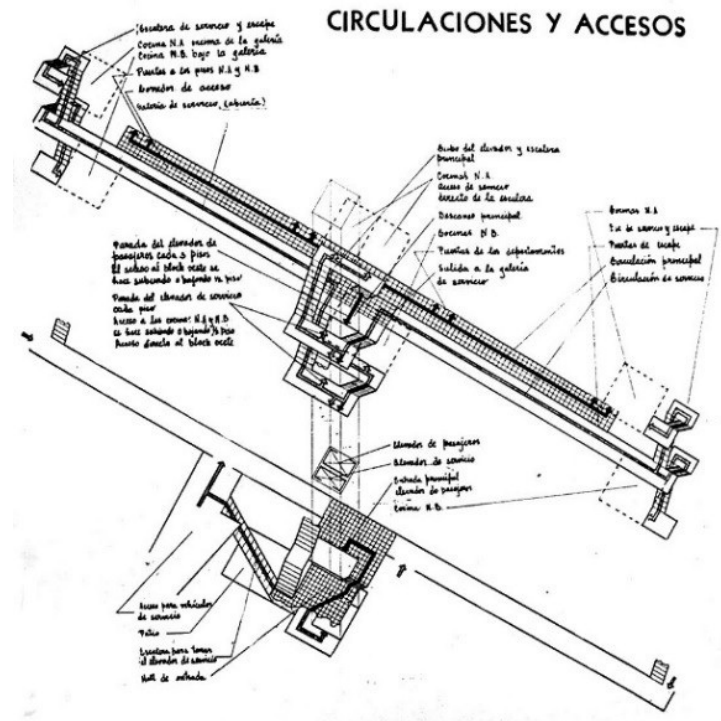

Fuente: Revista Arquitectura/México, julio 1939.
Adicionalmente, en el artículo publicado por Pani se mencionan diversas ventajas del uso del "tres en dos", como acabó traduciendo a la técnica. Entre éstas estaba la reducción de superficies de circulación y los costes de construcción, aunque también mencionaba la posibilidad de "flexibilidad" para generar varios tipos de departamentos, con diferente número de recamaras, una cualidad que Pani no llegará nunca a dominar. La flexibilidad es un aspecto potencial del 3-2 system de la cual especula Coates, mencionando que "podría llegar hasta 40 variantes diferentes" (Cantacuzino, 1978), aunque nunca llega a ponerla realmente en práctica ni a publicar la solución gráfica.

La percepción de estas singulares organizaciones domésticas en la época es descrita claramente por Xavier Moyssén $(2008 ; 127)$ :

(... ) este tipo de departamentos [de dos pisos] ya había sido propuesto con anterioridad [al CUPA] por Mario Pani, en edificios como los de Paseo de la Reforma 334 y de Río Balsas 36, [...] con la disposición de dos en tres (dos departamentos en tres pisos), que buscaba incrementar la intimidad del área de recámaras y emular la disposición de una casa, lo que por esas épocas constituía una opción privilegiada por los mexicanos.

A partir de la publicación de julio de 1939 que hemos comentado puede ser trazada, dentro de la obra de Pani, una línea de trabajo con gran preocupación por la distribución interna del edificio, con sensibilización, destreza e interés por generar un modelo nuevo de departamento desarrollando el proyecto en sección (usamos la expresión con la que el propio Coates definió la técnica), dentro del cual se agrupan proyectos de diferentes programas e intereses.

Según Pani, la idea de construir "con economía de espacio y con esas características de grandes edificios", como los de la Ville Radieuse de Le Corbusier y los multifamiliares, fue aplicada al realizar "algunos edificios altos en terrenos que entonces había", concretamente en Reforma 334 (...), una obra muy parecida a otro que aún está en pie, en la calle Balsas. Ahí experimentó con el concepto de dos en tres, es decir, dos departamentos en tres pisos, de manera que cada departamento tuviera un piso y medio en la estancia. El elevador se paraba en un nivel y medio y había dos puertas que eran para ir al nivel de abajo y al de arriba. La principal ventaja consistía en que el elevador no paraba en cada piso, sino en cada tres pisos" (Mereles, 2008, p. 364). 
En 1941, apenas dos años después de la publicación sobre el diseño 2-3 de Coates, Pani desarrolla un proyecto en 1941 para un edificio en la colonia Chapultepec, el cual representa una primera incursión en el uso del planeamiento en sección. El proyecto se desarrollaba dentro de una morfología diferente de la del Palace Gate y, en vez de utilizar un bloque laminar, Pani desarrolla una versión del 3-2 system dentro de una torre. Y si bien el resultado demuestra una admirable destreza geométrica, las ventajas son dudosas, pues no existe reducción significativa de área de circulación colectiva (el rellano de elevador se repite en todos los pisos, con ligeras variantes) ni tampoco existe posibilidad de generar flexibilidad.

No obstante, la organización conseguía dividir circulaciones de servicio/propietarios: el elevador podía abrir, en el caso de los pisos alto y bajo, directamente sobre el rellano de acceso a la cocina, donde existían adicionalmente dos pequeñas piezas en el lado opuesto a la cocina, llamados "criados" (lo que permite interpretar este espacio como un área exclusiva de servicio). En los pisos intermedios, el elevador abría sobre las puertas de acceso a los apartamentos.

La propuesta del proyecto en Chapultepec no llegó a ser construida, pero una versión similar fue construida en otro lote sobre la calle Balsas, además de ser publicada en la revista Arquitectura/México de 1945. Algunas variantes fueron introducidas con respecto al proyecto anterior, pues lo que eran los espacios "criados" vienen a ser substituido por una recámara adicional incorporada al departamento.

En particular, sobre este último proyecto, cabe observar algunos puntos atípicos de la estructura habitacional. El acceso de propietarios es realizado a través de un "vestíbulo", en el piso medio, que reparte para una zona de recámaras, estando en el piso inferior, o superior, la zona pública. Así, una particularidad aquí era que visitante pasaba, primeramente, frente a una zona de dormitorios para después llegar a la sala de estar y comedor. La organización era completamente inusual y en contra del sistema tradicional, con una zona pública inferior y la zona íntima en el piso superior protegida de las miradas indiscretas. Por otro lado, la sala de estar era un espacio vistoso, colocado medio piso por debajo (o por encima) del nivel de acceso, contando con una altura y media de entrepiso, inscrito dentro de un espacio en semicírculo. Desde el estar se abrían vistas sobre el paisaje urbano, estando los usuarios protegidos contra la entrada excesiva de sol gracias a los brise-soleils.
Figura 6. Departamentos en calle Balsas (1941).
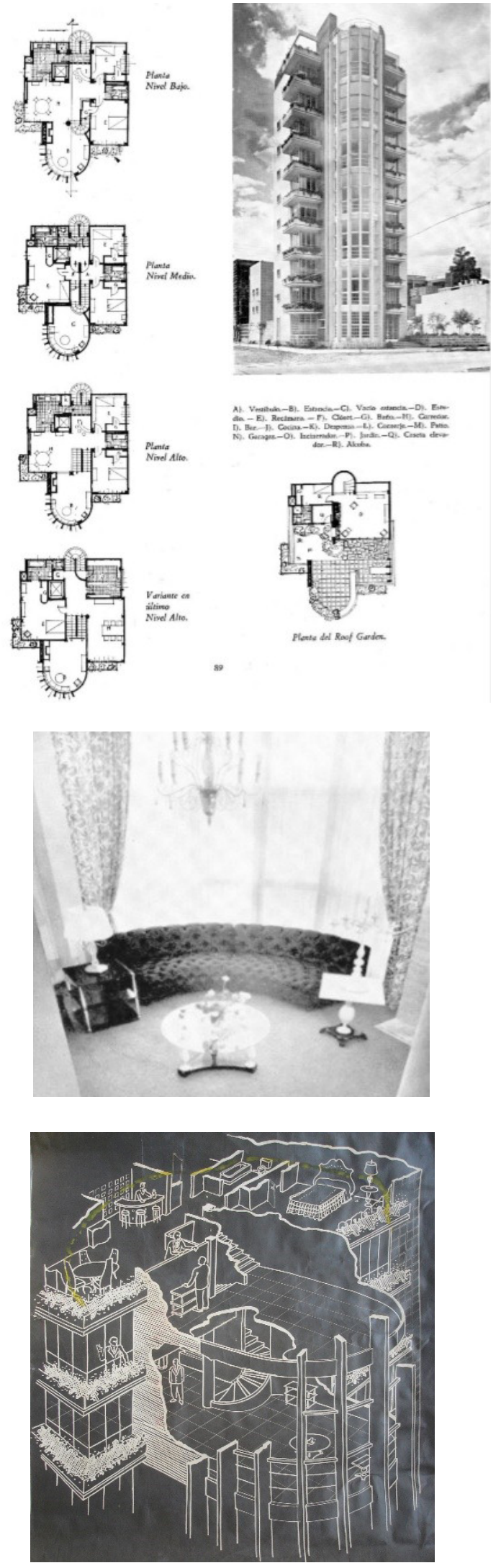

Fuente: revista Arquitectura/México, ene. 1945 /AAM-FA-UNAM. 
Otro proyecto de Pani de 1941 sobre la avenida Paseo de la Reforma 334 (hoy demolido), consiste en una torre entre medianeras, en la que aparece una organización similar al 3-2 system. Tampoco se observa en este caso tentativa alguna de aprovechar las posibilidades de reducción de circulación horizontal colectiva que incluso es completamente nula, ya que todos los pisos el rellano de la escalera y elevador aparecen exactamente con la misma superficie. Por el contrario, la atípica distribución con recámaras en el mismo piso del acceso vuelve aparecer.

Estas propuestas deben ser entendidas como experimentos; lo que Pani está desarrollando es un auténtico laboratorio de indagaciones, a través de un camino de prueba y error. La pregunta que parece subyacer aquí es cómo debe ser la habitación "moderna" y cómo adaptarla a una conservadora sociedad, reacia a vivir dentro de un modelo verticalizado de departamentos, con una importante inercia a mantener su forma de vida y la estructura espacial que le es más cara y más íntima: la casa familiar mexicana.

Figura 7. Departamentos en Av. Paseo de la Reforma 334 (1941), Mario Pani. (Continúa en columna derecha)

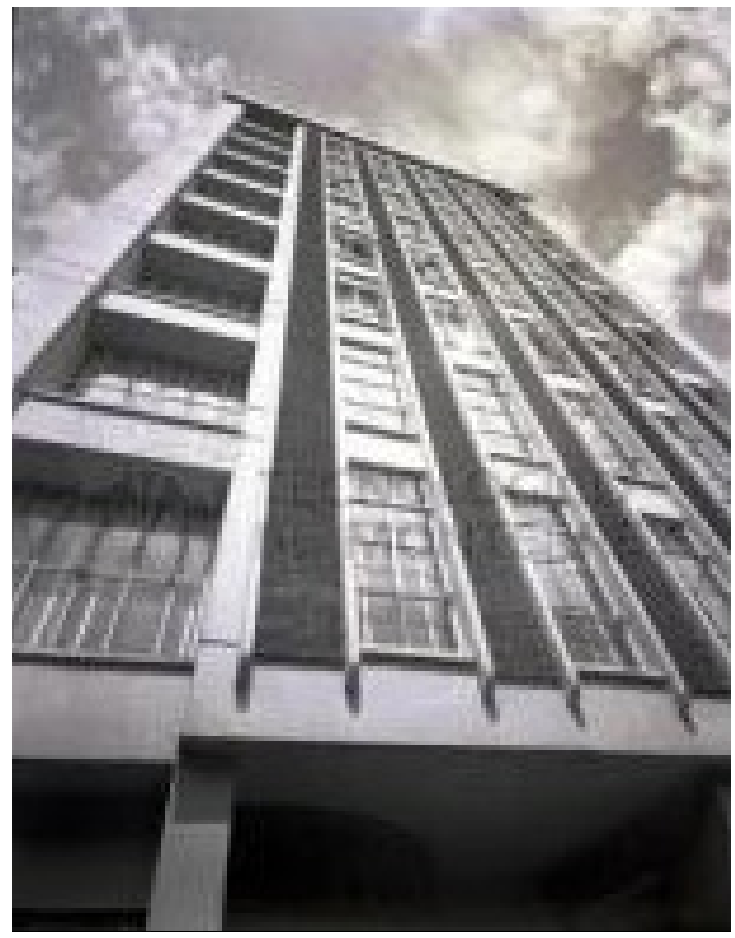

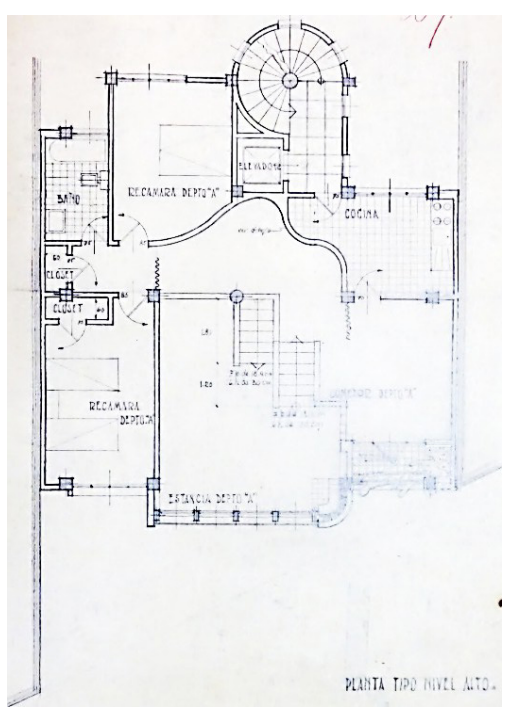
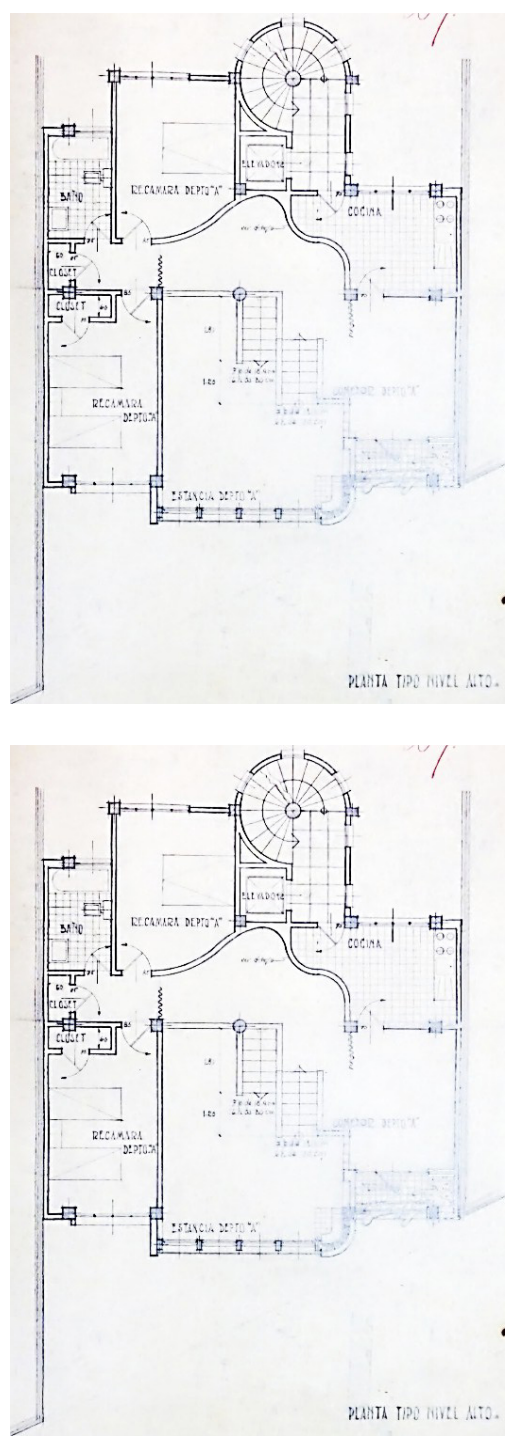

Fuente: Archivo de AAM-UNAM 
Después de la construcción del edificio Balsas detectamos una pausa en los experimentos proyectuales de Pani, y no existen registros de planos de habitación colectiva, al menos en su archivo personal conservado en el Archivo de Arquitectos Mexicanos-UNAM, hasta la construcción de los multifamiliares CUPA (1947-1949) y, posteriormente, el Conjunto Urbano Presidente Juárez -CUPJ (1950-52). Es interesante, en esta coyuntura, volver a observar lo que se estaba divulgado en los medios especializados, principalmente revistas de arquitectura extranjeras, pero que circulaban en México en publicaciones como Architectural Forum y Architectural Record (Pérez-Duarte, 2005) ${ }^{13}$. Dentro de éstas, en relación con modelos desarrollados con planeamiento en sección, existe un importante número de publicaciones que elucubran sobre posibles reducciones de costos de construcción. En términos generales, la argumentación (muchas veces apoyada en cálculos matemáticos y geométricos) colocaba en relación, generación de espacio privativo en oposición al espacio colectivo de circulación.

Un modelo llamado skip-stop despertaba entusiasmo $^{14}$ y frecuentemente se hablaba de éste con un optimismo de tal magnitud que las disquisiciones llegan a apuntar la posibilidad de ser utilizado a gran escala, de forma masiva para habitación de interés social ${ }^{15}$.

Y lo que estaba en la teoría en los años cuarenta parece consolidarse en los cincuentas, lo que explica la gran cantidad de conjuntos habitacionales con uso de planeamiento en sección, no solo en México, sino en todo el mundo occidental ${ }^{16}$. En este sentido, como insistentemente defendía Pani (Garay, 2000), el proyecto y la construcción del CUPA representa una propuesta pionera.

Particularmente, en relación a los departamentos del CUPA, Pani y Salvador Ortega (quien aparece como coautor de aquí en adelante en todos los proyectos habitacionales) proponen cinco tipologías, de las cuales las llamadas tipo "A" y "C" son las más comunes, y son sorprendentemente próximas al skip-stop, el cual no es asimilable al 3-2 system, pues no cuenta con espacios de altura y media de entrepiso, siendo considerado por ello una organización más eficiente.

Pani utiliza de hecho el modelo skip-stop posteriormente también en un proyecto denominado "Edificio multifamiliar para empleados del Hospital Colonia" (1951), en el cual los departamentos del "edificio B" muestran un corredor a cada tres pisos. El "Edificio A" del referido proyecto consiste, por el contrario, en un bloque laminar, pero con distribución distinta, apareciendo escaleras colectivas entre dos módulos de departamentos a los cuales da acceso, que a su vez conectan con un corredor colectivo a cada tercer piso; una configuración aún más eficiente, posteriormente llamada de sistema Verteilergängen (Zumpe, 1978).

\section{Figura 8. Multifamiliar para empleados del Hospital Colonia (1951), Mario Pani y Salvador Ortega. (Continúa en página 52)}

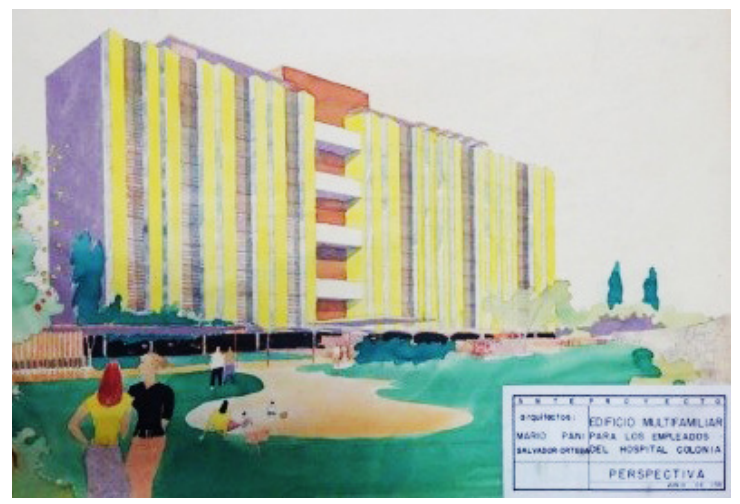

\footnotetext{
${ }^{13}$ En consultas en archivos personales de diversos arquitectos contemporáneos a Pani, se han encontrado varios ejemplares, algunas veces con anotaciones y recortes, síntoma de la intensidad con que eran observados estas publicaciones.

14 "Skip-Stop Elevators Permit Low Rent", Architectural Forum, set. 1946, "Eastgate- A New Plan Type". Architectural Record, Jan. 1949, Architectural Record, Jan. 1949, L'Architecture D'Aujourd'Hui, Aug. 1952 "High Apartments or Low", Architectural Forum, Jan. 1952, "Skip-Level Elevators and Duplex Apartments Cut Construction and Operating Costs", Architectural Forum, Jan. 1950.

${ }_{16}^{15}$ El conjunto Pruitt Igoe (St Louis, 1954), por ejemplo, respondía a una lógica de proyecto derivada de estas discusiones.

${ }^{16}$ En Londres, por ejemplo, el programa de London County Council incorporó, de manera casi sistemática, el uso de pisos alternativos, dando lugar a icónicos conjuntos como Golden Lane o Park Hill, contemporáneas al Robin Hood Gardens, de los Smithsons. En Suiza, el ejemplo de Schönberg (Freiburg, 1967) del programa Sicoop. En América Latina, en Brasil, el conjunto Japurá (São Paulo, 1948), otro ejemplo del programa de los I.A.P.I. En Venezuela, aparecen muchos ejemplos, como la hacienda Cerro Grande (1954) y la Unidad Quinta Crespo (1952).
} 


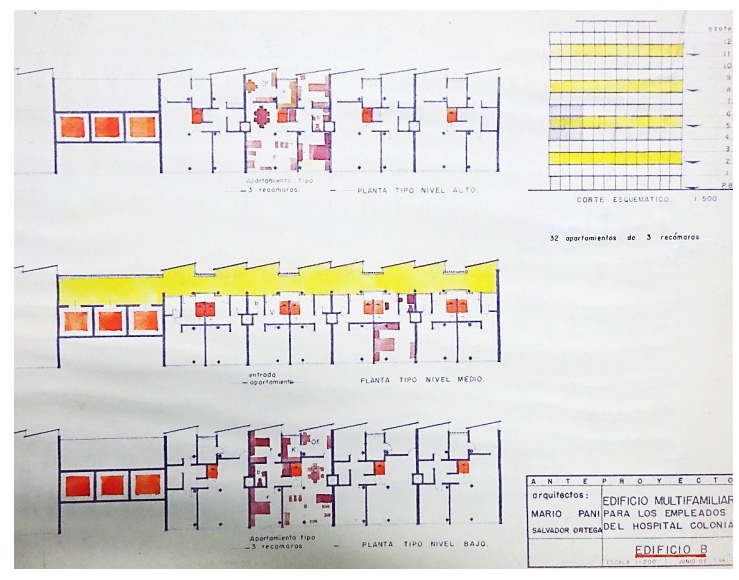

Fuente: AAM-UNAM.

Aquí la ventaja de utilizar un bloque laminar, y no torre, es evidente; se observa ahora sí que la disminución de superficie es significativa (en amarillo en el plano), lo cual llevaba directamente a un menos gasto de construcción, de acuerdo las discusiones mencionadas. No obstante, una distribución atípica sigue apareciendo aparece aquí, ya que el visitante era obligado a pasar frente al dormitorio principal antes de subir o bajar a la sala de estar.

La secuencia de proyectos comentados hasta ahora abren una discusión que puede explicar la aparente disminución de eficacia (la posible reducción de superficie de corredor colectivo) con el rescate de un modelo de dúplex más tradicional en CU. Pensamos que el dúplex utilizado para una distribución más conservadora en dos plantas (invariablemente con el acceso y zona pública abajo, zona íntima arriba) mostraba mayor proximidad con la casa unifamiliar tradicional, siendo el modelo que acabará siendo utilizado en CU.

El dúplex, representa de cierta forma, un encuentro entre fuerzas opuestas. Por lado, la modernidad funcional, lógica y racional, que reduce costes de construcción con innovadoras y atípicas formas habitacionales. Por otro lado, la tradición, que busca conservar los valores y organizaciones ancestrales, recogidos en la casa unifamiliar histórica. Y es interesante recordar que, desde la década de los treinta, venia consolidándose una forma muy extendida conocida como "casa de hall"; una casa unifamiliar compacta de dos niveles, organizada alrededor de un espacio central de recepción y representación, con zona pública abajo y zona íntima escaleras arriba (Cruz 2015: 556). Pani coloca en práctica el dúplex en un an- teproyecto en 1947, con pequeñas células de apenas $84 \mathrm{~m} 2$ (42 m2 por piso), denominado "Departamento tipo para 8 personas" -aunque presenta una célula con capacidad máxima de 6 personas.

El proyecto consiste en tres bloques laminares, en implantación viaria, organizados mediante un corredor a cada dos niveles. Sin buscar profundizar mucho en la curiosa distribución interna, sí es significativo señalar una lógica más próxima a la tradición de la casa unifamiliar: una zona pública en la parte inferior, donde se accede, y la planta superior "recámaras", escalera arriba. En la parte superior se puede observar la proyección de la planta por encima de corredor colectivo que, -sin llegar a la fachada, -para protección de sol quizá- gana superficie privativa. La propuesta parece conciliar, por lo tanto, tradición con la eficiencia "moderna".

Figura 9. Departamentos para 8 personas (1947), Mario Pani.
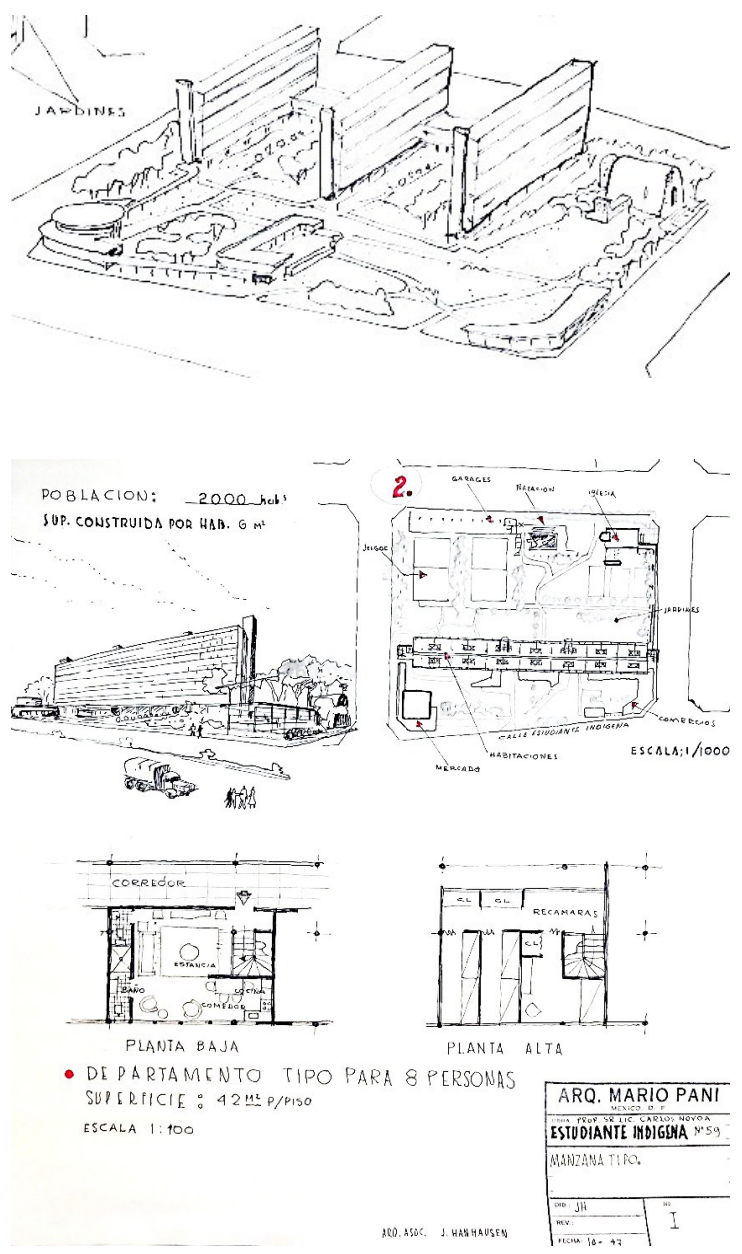

Fuente: AAM-UNAM. 
Una distribución del dúplex se observa también posteriormente en el CUPJ, en el edifico "C", donde aparecen células en dos niveles, con dos versiones de 3 recámaras (siendo una de estas una alcoba) y de 2 recámaras, esta última dentro de un módulo más estrecho, colocadas estratégicamente entre los dos núcleos de escalera para reducir superficie del corredor colectivo.

En una de las fachadas, los corredores aparecían protegidos por una celosía, aunque eran innecesarios para protección del sol (reciben orientación noroeste), aunque generaban una potente imagen, con franjas en sombra que aparecían al observar el inmueble desde la calle; no por otra razón los edificios "C" se localizaban sobre el eje de la avenida Orizaba, de forma perpendicular. Se trataba de una apariencia generada desde la lógica de la eficiencia, una circulación horizontal que se reducía a la mitad colocando en práctica la distribución en dúplex.

Figura 10. Departamentos de los bloques tipo "C" del CUPJ.

(Continúa en columna derecha)
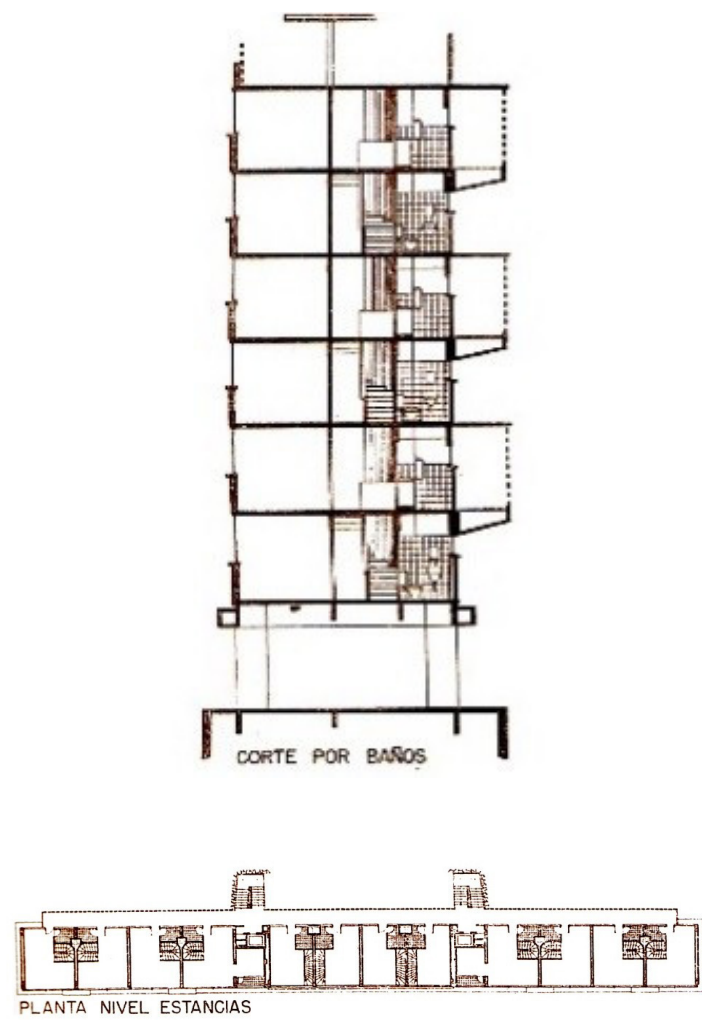
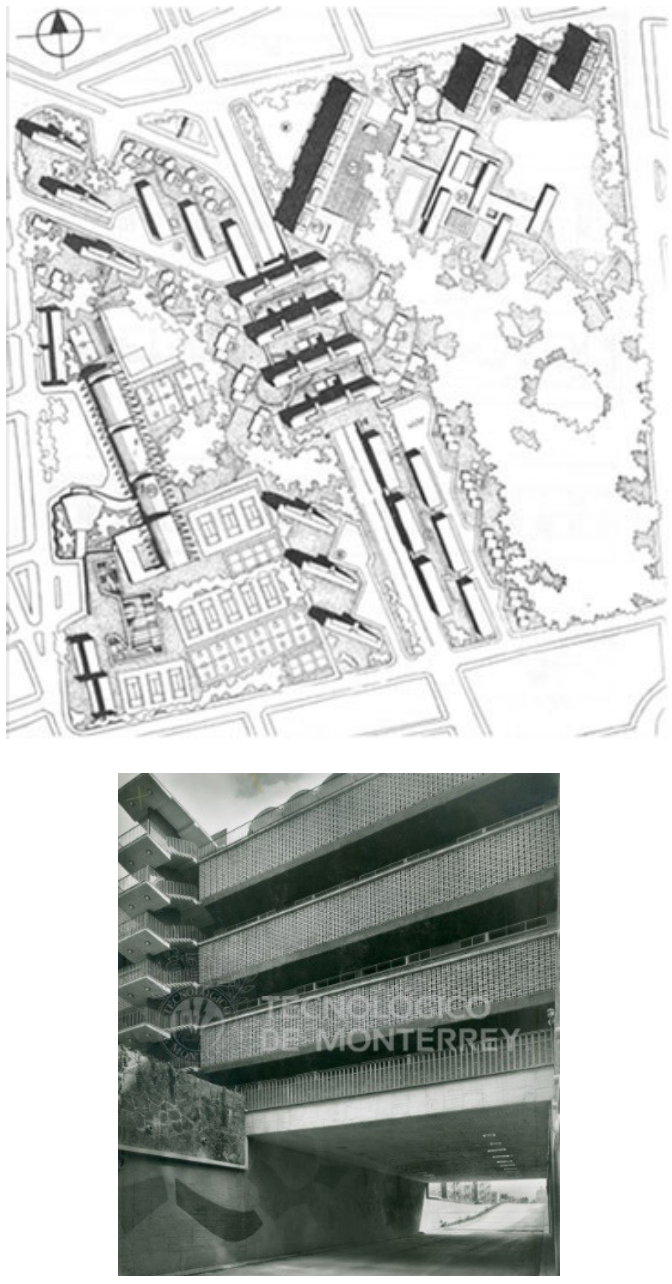

Fuente: Pani,1952; fotografía de la Colección Mario Pani, Patrimonio Cultural del Tecnológico de Monterrey.

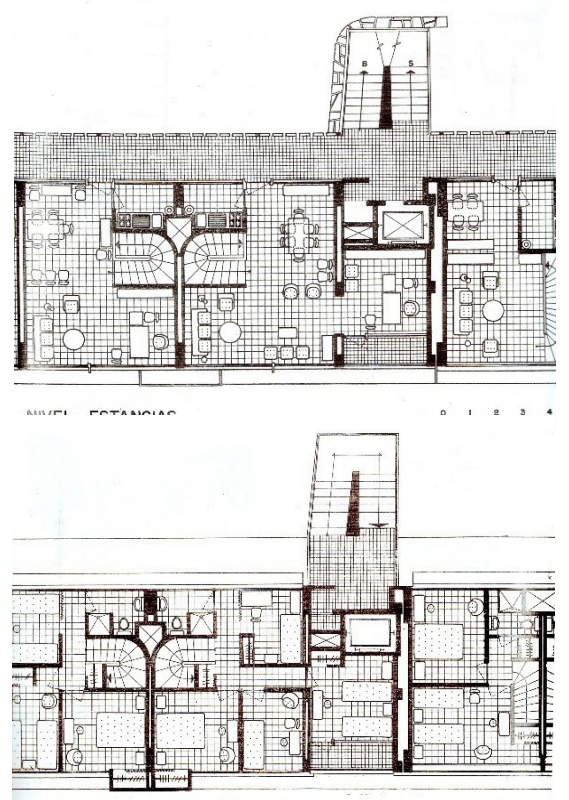

NIVEL RECAMARAS

Fuente: Pani, 1952. 
El multifamiliar está compuesto por un bloque laminar, dejando $20 \%$ del terreno libre, e implantado de acuerdo con el eje heliotérmico norte-sur, como la Unité de Marseille, recibiendo sol en "la totalidad de las habitaciones que ven al oriente y al poniente". Pensado específicamente como un modelo "multifamiliar para maestros", la propuesta aloja 35 viviendas asimilables a la estructura de una casa familiar, incluyendo en su programa 42 células, teniendo apenas 10 tipo "estancia-alcoba", para solteros o recién casados, dispuestos en la planta baja y basamento. Es en los niveles superiores donde aparecen los restantes 32 departamentos, una apuesta que muestra una búsqueda de equilibrar innovación y tradición. Una concesión a la tradición importante aquí es el uso de un modelo más conservador de "departamentos de dos pisos", que coherentemente estructuran la zona pública "en el nivel de la entrada, la estancia, comedor y cocina", opuesto a la zona íntima superior de "dos recámaras, alcoba y baño".

No obstante, es interesante aquí observar una particularidad en relación con la organización vertical en un anteproyecto del multifamiliar de CU, datado en 1950. Sucede que en la primera versión del dúplex se puede observar una curiosa disposición: el acceso es realizado por el piso superior, y no el inferior, donde está la zona pública, estando la zona intima abajo. La inversión de posición entre zonas era completamente extraña al orden común en cualquier casa o modelo dúplex. Un caso similar con inversión de dúplex se puede encontrar también en un pequeño edifico de cinco niveles de Enrique del Moral (cfr. Arquitectura/ México, ene.1945).

Figura 11. Detalle del dúplex del anteproyecto del multifamiliar para maestros en CU, 1950.

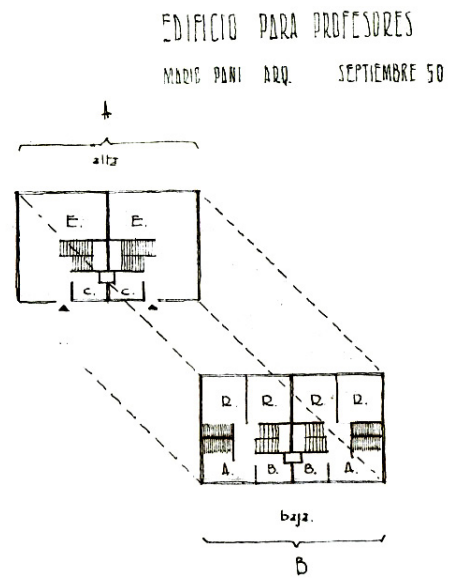

Fuente: Dirección General de Obras y Conservación, UNAM.
La explicación de esta disposición atípica responde al ánimo experimental de la época que hemos comentado. Si se consultan manuales de la época y recopilaciones de edificios de departamentos, el ruido representaba una de las principales objeciones en contra de vivir en un departamento. La molestia, sin embargo, podía ser evitada con una organización estratégica (Kamenka, 1947: 111).

En el manual de Yorke y Gibberd, que tuvo numerosas reediciones, anotaban que el dúplex convencional era "malo desde el punto de vista del ruido, puesto que la sala del apartamento está inmediatamente sobre las piezas destinadas a dormir del vecino", pero se puede resolver con un planeamiento adecuado: "el tipo maisonette (dúplex, en inglés británico) debería alternarse en la sección para superponer las salas y dormitorios" proporcionando así "la gran ventaja de que las zonas de silencio son adyacentes" (Yorke y Gibberd, 1948: 24). Así, es posible que el anteproyecto respondiese a preocupaciones acústicas: el ruido generado en zona publica del vecino, queda siempre amortiguado por la inserción de un nivel intermedio de zona de silencio (las recámaras).

A pesar de las posibles ventajas acústicas, la versión final acabó utilizando un modelo más conservador de dúplex, con acceso y zona publica inferior, y subiendo la escalera, las recámaras. La organización dúplex, no obstante, fue capaz de generar una innovadora imagen de modernidad pues "de esta manera, las circulaciones horizontales se reducen a una por cada dos en las que, también se para el elevador", y que se refleja en la fachada -igual que en el edificio "C" de CNPJ.

(...) la unidad de circulaciones [...] se acusa en la fachada Poniente por medio de grandes celosías de block Pirámide; contrastan agradablemente con los muros cerrados del nivel de las recámaras, tratados con vitricotta en colores marrón y almendra [y en la fachada opuesta] al Oriente, los balcones de las estancias, con muros bajos de concreto, se alternan cada dos niveles, formando un dibujo rítmico. (Arquitectura/México, sep. 1952). 
Figura 12. Versión final construida del multifamiliar en CU.
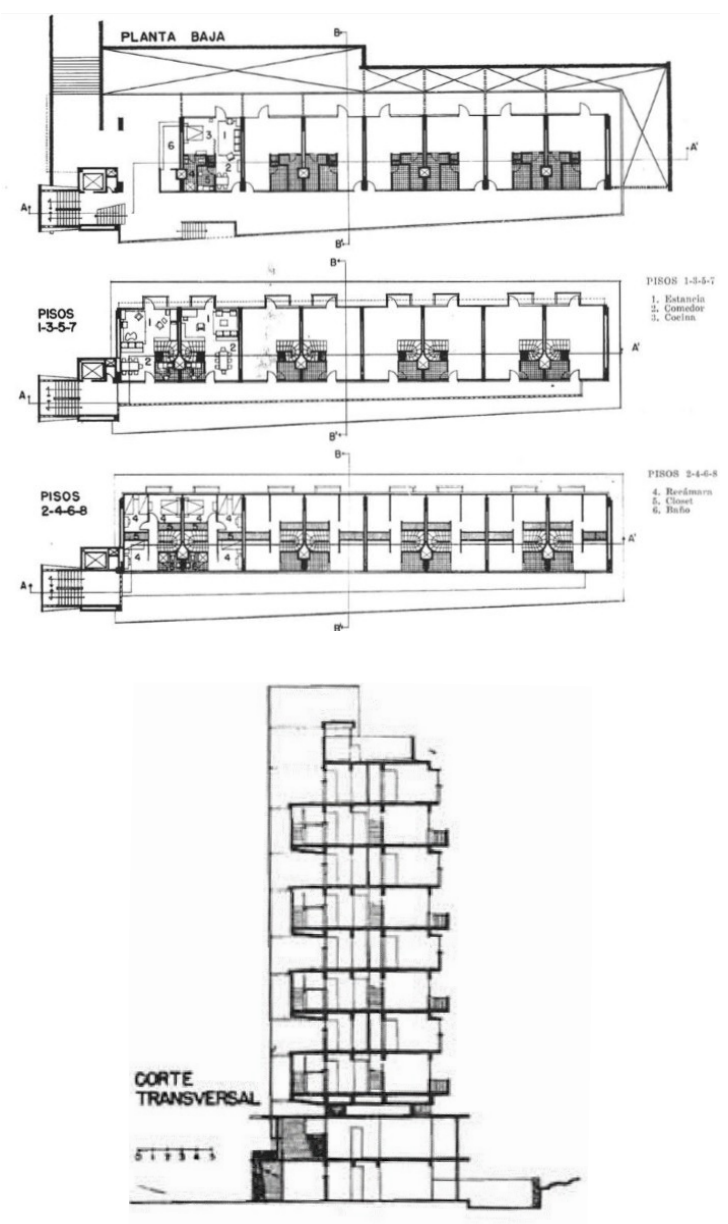

Fuente: Revista Arquitectura/México. sep. 1952.

El proyecto para el Multifamiliar de CU se encuentra al final de una experiencia de poco más de una década, en la que se observa una evolución, con Pani inicialmente buscando poner en práctica modelos en la línea con la más rabiosa innovación. En el inicio de los cuarenta se observa un ímpetu que parece madurar con el transcurso del tiempo, concluyendo en formas que se acercan más a la tradición doméstica mexicana. Hemos visto que el dúplex del Multifamiliar en CU remite a una estructura con la cual la población se encontraba ya familiarizada. Así pues, no es sorprendente que, en el momento de realizar Pani la propuesta para el Multifamiliar de CU, se observe prudencia, o quizá cierta sabiduría adquirida después de toda la búsqueda de los años cuarenta. La modernidad, lógica y racional, no se impone de forma insensible, sino ahora respetaba el sistema de privacidad familiar. $\mathbf{C}$

\section{Referencias bibliográficas.}

Adriá, M. (2005). Mario Pani: la construcción de la modernidad. México, Conaculta/GG.

De Anda, E. X (2008), Vivienda colectiva de la modernidad en México. Los multifamiliares durante el periodo presidencial de Miguel Alemán (1946-1952). México, iie-unam.

Cantacuzino, S. (1978). Wells Coates. Londres, Gordon Fraser.

Cruz González Franco, L. (2015). "Los espacios de vivienda", en Chanfón Olmos, Carlos (coord.). Historia de la arquitectura y el urbanismo mexicanos. Vol. IV. Tomo II. México, FCE.

Del Moral, E. (1964). "Ensayo sobre el Estilo". En Cuadernos de Arquitectura INBA núm. 16, diciembre. Garay Arellano, G. (2000). Mario Pani: Historia oral de la Ciudad de México, testimonios de sus arquitectos (1940-1990). México, CONACULTA/ Instituto Mora.

Gómez Porter, P. (2016). "La construcción de los multifamiliares de Mario Pani: historia, problemas y retos actuales". Boletín de monumentos históricos, Tercera época, núm. 36, enero-abril.

Langenscheidt, E. (1965). "La Habitación residencial en México". Arquitectura México núm. 91 (septiembre d), p. 137.

Larrosa, M. (1985). Mario Pani, arquitecto de su época. México, UNAM.

Mereles Gras, L. (2008). "Entrevista a Mario Pani", en Noelle, Louise (comp.). Mario Pani. UNAM-IIE, México.

Kamenka, H. (1947). Flats: Modern Developments in Apartment House Construction, Londres, Crosby Lockwood \& Son.

s/a (1952). "Multifamiliar para maestros". Arquitectura México núm. 39, septiembre 1952.

s/a. Skip-Stop Elevators Permit Low Rent", Architectural Forum, set. 1946.

s/a. "Eastgate- A New Plan Type". Architectural Record, Enero 1949.

s/a. Architectural Record, enero 1949.

s/a. L'Architecture D'Aujourd'Hui, Agosto 1952.

s/a. "High Apartments or Low", Architectural Forum, Jan. 1952.

s/a. "Skip-Level Elevators and Duplex Apartments Cut Construction and Operating Costs", Architectural Forum, Jan. 1950.

Moyssén, X. (2008). "El Centro Urbano Presidente Alemán". En Noelle, Louise (comp.). Mario Pani. México, UNAM-IIE. 
Pani, M. (1952). Los multifamiliares de pensiones. México, Editorial Arquitectura.

Pérez-Duarte, A. (2005). La instauración del apartamento moderno: Ciudad de México 1925-1056. Tesis de doctorado en la UPC, Barcelona, 2005.

Pérez-Méndez, A. (2014). "Conceptualización de la ocupación del Pedregal" En Habitar CU 60 años. México, UNAM, 2014.

Sánchez Michel, V. (2014). Construcción de una utopia: Ciudad Universitaria, 1928-1952. Tesis de Doctorado. México, El colegio de México.

Sonderénguer C., P. (2008). "El primer edificio de condominio en México". En Noelle, Louise (comp.), Mario Pani. México, UNAM-IIE.

Yorke, F. R. S. y Gibberd, F. (1948). The Modern Flat. Londres, Architectural Press.

Zumpe, M. (1967). Wohnhochhäuser. Berlín, VEB Verla für Bauwessen.

\section{Imágenes}

Archivo Arquitectos Mexicanos UNAM (AAMUNAM): colección Mario Pani.

Archivo de la Dirección General de obras y Conservación UNAM (ADGOC).

Archivo aerofoto-ICA.

Archivo Histórico de la UNAM, Fondo UNAM, Secretaria General, caja 324, expediente 1833, 1951-noviembre (Entre la Dirección de Pensiones Civiles e ICA). "Contrato de obra a precio alzado." AHUNAM, Fondo UNAM, Secretaria General, caja 324, expediente 1833, 1953-octubre (De la Asociación de profesores universitarios al rector Dr. Nabor Carrillo). "Memorándum sobre el problema de organización de las Habitaciones de Estudiantes en la CIUDAD UNIVESITARIA". AHUNAM, Fondo UNAM, Secretaria General, caja 324, expediente 1833, 1953-marzo (De la Asociación de profesores universitarios al rector Dr. Nabor Carrillo). "Solicitud para apartar terrenos".

AHUNAM, Fondo UNAM, Secretaria General, caja 324, expediente 1833, 1953-julio (De Roberto Ramos, Consejero de empleados ante el $\mathrm{H}$. Consejo Universitario, al Rector Dr. Nabor Carri1lo). "Solicitud de terrenos para sus hogares dentro o cerca de la Ciudad Universitaria."

AHUNAM, Fondo UNAM, Secretaria General, caja 324, expediente 1833, 1953-octubre (De la Asociación de profesores universitarios al rector Dr. Nabor Carrillo). "Memorándum sobre el problema de organización de las Habitaciones de Estudiantes en la CIUDAD UNIVESITARIA".
Ver AHUNAM, Fondo UNAM, Secretaria General, caja 324, expediente 1833, 1954-julio (Del arquitecto Cesar Novoa al rector Dr. Nabor Carrillo). "Habitaciones de profesores y empleados en C.U." 